\title{
Borderline hypoplasia of the left ventricle in neonates: Insights for decision-making from functional assessment with magnetic resonance imaging
}

Lars Grosse-Wortmann, MD, ${ }^{\text {a,b }}$ Tae-Jin Yun, MD, ${ }^{\mathrm{a}}$ Osman Al-Radi, MD,${ }^{\mathrm{a}}$ Siho Kim, MD, ${ }^{\mathrm{a}}$ Masaki Nii, MD, ${ }^{\mathrm{a}}$ Kyong-Jin Lee, MD, ${ }^{\mathrm{a}}$ Andrew Redington, MD, ${ }^{\mathrm{a}}$ Shi-Joon Yoo, MD, ${ }^{\mathrm{a}}$ and Glen van Arsdell, MD ${ }^{\mathrm{a}}$

Objectives: We sought to compare the usefulness of echocardiography and magnetic resonance imaging in neonates with a borderline small left ventricle.

\begin{abstract}
Methods: The preoperative magnetic resonance and echocardiography studies of 20 consecutive patients (mean age $10 \pm 9$ days) undergoing magnetic resonance imaging were analyzed. The diagnoses were aortic stenosis $(\mathrm{n}=3)$, hypoplastic left heart complex $(\mathrm{n}=12)$, and unbalanced atrioventricular septal defect $(\mathrm{n}=5)$. The magnetic resonance imaging protocol included ventricular volumetry, flow measurements, and angiography. Potential left ventricular volumes, assuming an ideal geometric shape, were calculated by mathematically " unfolding" the compressed left ventricle.
\end{abstract}

Results: Left ventricular end-diastolic volume was $16.0 \pm 7.0 \mathrm{~mL} / \mathrm{m}^{2}$ of body surface area by echocardiography and $33.5 \pm 15.5 \mathrm{~mL} / \mathrm{m}^{2}$ by magnetic resonance imaging. Echocardiography consistently underestimated left ventricular volume and did not correlate with magnetic resonance. Of all echocardiographic parameters, mitral valve z-score was the best predictor of left ventricular end-diastolic volume by magnetic resonance $(r=0.77$; $P=.02$ ). The average potential volume increase was $8.8 \%$ for aortic stenosis, $35.0 \%$ for atrioventricular septal defect and $23.0 \%$ for hypoplastic left heart complex patients. Aortic valve diameter did not correlate with flow volume in the ascending aorta. Sixteen $(80 \%)$ of 20 patients underwent biventricular repair, without early mortality. Of these, only $5(31.3 \%)$ had a preoperative left ventricular end-diastolic volume of more than $20 \mathrm{~mL} / \mathrm{m}^{2}$ by echocardiography.

Conclusions: Magnetic resonance imaging is feasible in neonates with borderline left ventricular hypoplasia. Echocardiography does not accurately measure left ventricular hypoplasia in these patients and may unfairly preclude some patients from a biventricular repair in whom magnetic resonance is reassuring.

Almost 50 years ago Noonan and Nadas $^{1}$ coined the term "hypoplastic left heart syndrome," referring to a spectrum of cardiac anomalies characterized by varying degrees of underdevelopment of the left heart and aortic arch. At the severe end of the spectrum, the aortic valve (AV) is atretic, the mitral valve (MV) is severely hypoplastic, and the diminutive left ventricle (LV) shows endocardial fibroelastosis. At the mild end, hearts with small, but not intrinsically stenotic AVs and MVs and without endocardial fibroelastosis have been termed "hypoplastic left heart complex (HLHC).,"2 That spectrum, however, is dichotomized by the binary surgical decisions of biventricular (BV) versus univentricular (UV) repair made early in life. The consequences of the wrong decision, if made toward a BV repair, are often detri-

From the Labatt Family Heart Center at The Hospital for Sick Children, The University of Toronto, Toronto, Canada ${ }^{a}$ and the Department of Pediatric Cardiology, RWTH University of Aachen, Aachen, Germany. ${ }^{\mathrm{b}}$

Received for publication Dec 25, 2007; revisions received March 10, 2008; accepted for publication April 13, 2008

Address for reprints: Shi-Joon Yoo, MD, Department of Diagnostic Imaging, The Hospital for Sick Children, 555 University Ave, Toronto, Ontario, Canada M5G

1X8 (E-mail: shi-joon.yoo@sickkids.ca).

J Thorac Cardiovasc Surg 2008;136:1429-36

$0022-5223 / \$ 34.00$

Copyright (c) 2008 by The American Association for Thoracic Surgery

doi:10.1016/j.jtcvs.2008.04.027 mental in the short term, whereas unnecessary selection toward a UV course may have adverse long-term functional outcomes.

We recognized the shortcomings of echocardiography, which is the main diagnostic tool in assessing the adequacy of the LV to support the systemic circulation after the BV repair. We hypothesized that preoperative functional magnetic resonance imaging (MRI) is feasible in neonates with borderline hypoplasia of the LV and would aid in the decision between UV and BV repair.

This report summarizes our initial experience in 20 patients with a small LV as measured by echocardiography who underwent MRI before their first intervention.

\section{METHODS}

Between March 2003 and August 2006, 20 consecutive patients with borderline hypoplasia of the LV and in whom there was controversy regarding the decision between $\mathrm{BV}$ and $\mathrm{UV}$ repair underwent a preoperative cardiac MRI. All patients had an LV end-diastolic volume (LVEDV) of less than $30 \mathrm{~mL} / \mathrm{m}^{2}$ of body surface area by the Simpson volumetric analysis and none of them had obvious cardiovascular anatomy demonstrated on echo that precluded a BV approach. There were three distinct groups: (1) patients with intrinsic critical AV stenosis (AS, $n=3$ ), (2) patients with structurally normal MV and AV and hypoplastic arches, grouped as HLHC, with or without coarctation $(n=12)$, and (3) patients with unbalanced atrioventricular septal defects (AVSD, $n=5)$. All studies were 


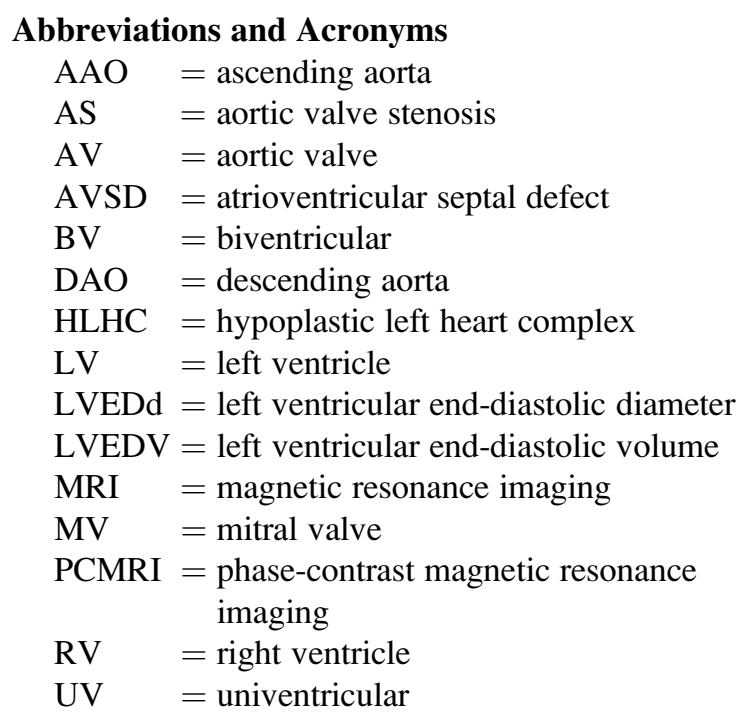

performed on a 1.5-Tesla scanner (GE signa CV/i; General Electric Medical Systems, Milwaukee, Wis) using a head coil. All but one patient were intubated during the study. Ventricular volumes and flow volumes were calculated on a commercially available work station (Mass Analysis and CV Flow; MEDIS Medical Imaging Systems, BV, Leiden, The Netherlands). In patients with AVSD, LV volumes were measured by MRI and echocardiography by virtually extending the deficient interventricular septum to the base of the heart.

The MRI protocol consisted of cine imaging in axial, 2-chamber, 4chamber, and short-axis planes, through-plane phase-contrast imaging (PCMRI) of the main, right, and left pulmonary arteries, ascending aorta (AAO) at the level of the right pulmonary artery, descending aorta (DAO) at the diaphragm, patent ductus arteriosus, superior vena cava, and atrioventricular valves, and contrast-enhanced MRI angiography.

\section{True and Potential LV Volumes}

The short-axis cine imaging for ventricular volumetry (Figure 1) was performed by using a segmented spoiled gradient refocused echo sequence with minimum echo and repetition times, flip angle $20^{\circ}$, bandwidth 31.25 $\mathrm{kHz}$, number of excitations of 2, slice thickness 3.6 to $5.0 \mathrm{~mm}$, number of slices 5 to 11 without gap, minimum field of view (160-200 mm), matrix $256 \times 160,20$ reconstructed phases per cardiac cycle.

Phoon and Silverman ${ }^{3}$ introduced the model of observed versus potential preoperative volume to highlight the concept that the LV may be underfilled and compressed by the volume-loaded right ventricle (RV), leading to an underestimation of its potential postoperative volume (Figure 2). Potential LV volume was defined as the expected volume when the crescentic LV is mathematically converted to an ellipsoid chamber. This is the expected shape after effective relief of RV overload and adequate LV filling. We applied this method to MRI volumetry. The endocardial circumference (C) of the LV cavity was measured in end-diastole in each short-axis plane. Assuming that $\mathrm{C}$ would be maintained after conversion of the crescentic to a round configuration, the potential LV area in each short-axis plane (Apot) and potential LV volume $\left(\mathrm{V}_{\text {pot }}\right)$ were calculated by using the following formulas:

$\mathrm{A}_{\text {pot }}=\mathrm{C}^{2} / 4 \pi$

$\mathrm{V}_{\text {pot }}=\sum \mathrm{A}_{\text {pot 1 n }} \mathrm{X}$ slice thickness

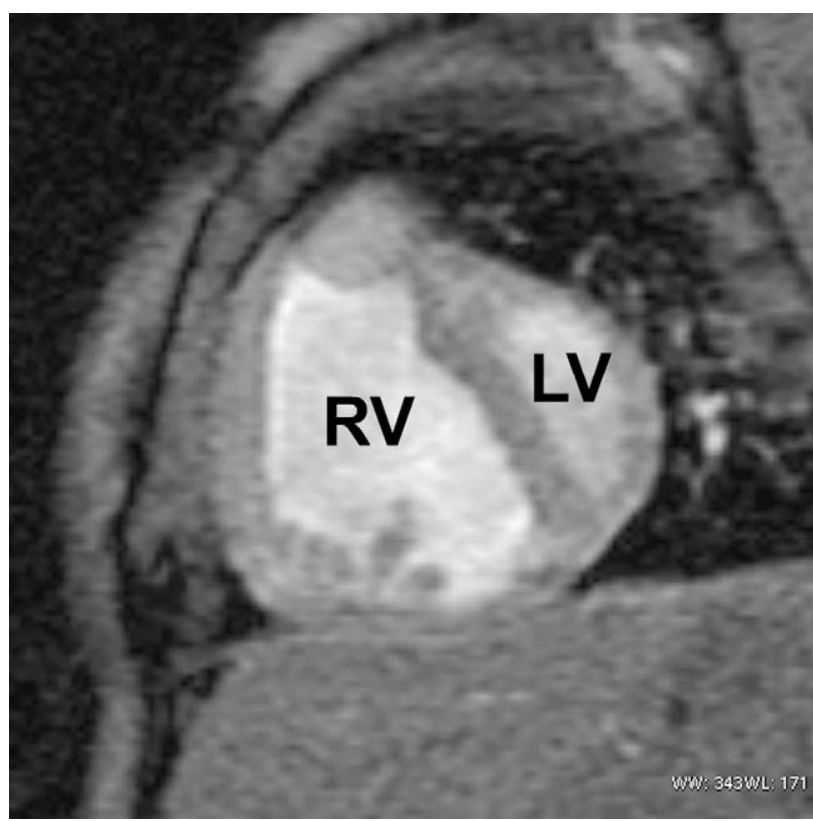

FIGURE 1. Short-axis slice at end-diastole in a patient with unbalanced atrioventricular septal defect. The left ventricle $(L V)$ is compressed by the right $(R V)$. The left atrioventricular valve is suspended by a single papillary muscle.

\section{Blood Flow}

Imaging parameters for PCMRI were as follows: Minimum echo and repetition times, flip angle $20^{\circ}$, bandwidth $31.25 \mathrm{kHz}, 1$ to $2 \mathrm{k}$-space lines per segment, number of excitations 2 , slice thickness $4 \mathrm{~mm}$, minimum field of view (160-200 mm), matrix $256 \times 160$, velocity encoding $150 \mathrm{~cm} / \mathrm{s}, 20$ reconstructed phases per cardiac cycle. Pulmonary blood flow was calculated by summating the right and left pulmonary arterial flow. Systemic blood flow was calculated by adding the flow volume to the upper compartment, represented by the superior vena cava flow, to the flow volume to the lower compartment, represented by the blood flow through the DAO.

\section{Angiography}

Magnetic resonance angiography was performed with suspended ventilation if tolerated by the patient's condition. A 3-dimensional fast spoiled gradient refocused echo sequence in the coronal orientation was used
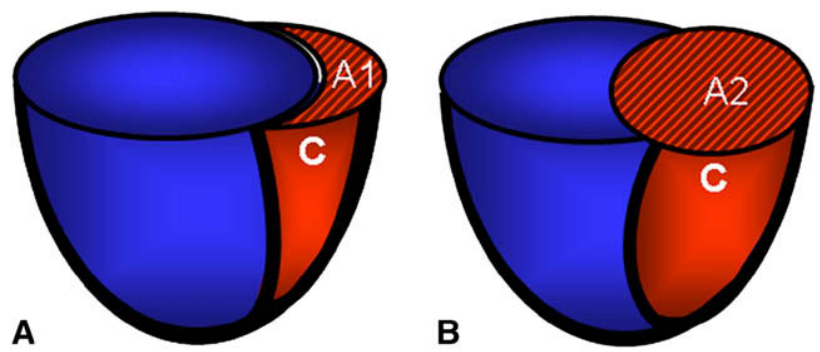

FIGURE 2. Observed (A) and potential (B) left ventricular volumes: Assuming that the left ventricle is compressible but otherwise noncompliant, the endocardial circumference $(C)$ in any short-axis plane should remain constant, irrespective of the filling state, whereas the encircled areas $\left(A_{1}\right.$ and $A_{2}$ ) depend on the shape of the left ventricle. The potential volume can be calculated from the area and the thickness of the imaging slices. 
with the following parameters: Minimum echo and repetition times, flip angle $30^{\circ}$, bandwidth $31.25 \mathrm{kHz}, 33$ to 45 partitions, slice thickness 1.5 to 3.0 $\mathrm{mm}$, minimum field of view (160-200 mm), matrix $256 \times 128,33$ to 45 partitions. A bolus of $0.2 \mathrm{mmol} / \mathrm{kg}$ dimeglumine gadopentetate (Magnevist; Berlex Laboratoires, Quebec, Canada) was injected manually through an antecubital or hand vein, followed by a saline chaser. Centric ordered data acquisition was set to start 6 seconds after the contrast medium reached the DAO by using an automated bolus tracking technique. The diameters of the AAO, transverse aortic arch, aortic isthmus, DAO, and patent ductus arteriosus were measured on the reconstructed images using maximum intensity projections.

Echocardiography was performed on one of the following machines: Hewlett-Packard Sonos 5500 (Agilent; Andover, Mass), ATL 5000 (Advanced Technology Laboratories, Seattle, Wash), Vivid 7 (General Electrics Medical Systems, Milwaukee, Wis), IE33 (Philips Medical Systems, Bothell, Wash). Measurements were partly obtained off-line and included LV end-diastolic diameter (LVEDd) by M-mode in the parasternal longaxis view, the diameters of the AV, MV, and tricuspid valve annuli in the parasternal long-axis and apical 4-chamber views, the diameters of the AAO, transverse aortic arch, aortic isthmus, DAO, and patent ductus arteriosus from suprasternal. Echocardiographic morphologic measurements were standardized to body surface area as z-scores on the basis of published normative data. 4

LVEDV was estimated by the Simpson rule. ${ }^{5}$ Postoperative measurements included the diameters of the AV and MV annuli in parasternal long-axis and apical 4-chamber views, respectively, as well as the LVEDd.

\section{Statistical Analysis}

The MRI and echocardiographic data were analyzed following approval by the institution's ethics board. Continuous variables are presented as mean \pm standard deviation and range. Proportions are presented as a frequency (percent). Univariable associations between continuous variables were assessed by the Spearman rho rank correlation test. Where appropriate, multivariable ordinary least square linear regression was used to assess preoperative echocardiographic predictors of the observed preoperative LVEDV by MRI. Serial echocardiographic measurements are presented using longitudinal data analysis plotting techniques. Moving average (Loess) curves of longitudinal data was done where appropriate with a smoothing span parameter $(\alpha)$ of 0.75 for the weighted least square method. ${ }^{6}$ All statistical analyses were done using the R statistical Package, ${ }^{7}$ using the Design ${ }^{8}$ and $\mathrm{NLME}^{9}$ libraries.

\section{RESULTS}

The patients' mean ages, weights, and body surface areas at the time of MRI were 10 days (2-37 days), $3.23 \mathrm{~kg}$ (1.70$4.40 \mathrm{~kg})$, and $0.21 \mathrm{~m}^{2}\left(0.14-0.25 \mathrm{~m}^{2}\right)$, respectively. The mean study time was 62 minutes and there were no complications during any of the studies. The interval between the echocardiogram and the MRI ranged from 1 to 15 days (mean of 5 days) The preoperative LVEDV measured by echocardiography ranged from 6 to $25 \mathrm{~mL} / \mathrm{m}^{2}$ of body surface area by the monoplane $(\mathrm{n}=20)$ and 10 to $30 \mathrm{~mL} / \mathrm{m}^{2}$ by the biplane Simpson method $(\mathrm{n}=8)$. The MV and AV $\mathrm{z}$-scores were $-4.0 \pm 2.0(-6.8$ to -0.2$)$ and $-4.6 \pm 2.2$ $(-8.6$ to -0.1$)$, respectively. A ventricular septal defect was present in 2 of the HLHC patients.

\section{Comparison Echocardiography-MRI}

Applying the monoplane Simpson method for echocardiographic volumetry (Figure $3, A$ ), the echocardiographic and MRI LVEDV measurements were $16.0 \pm 7.0 \mathrm{~mL} / \mathrm{m}^{2}$ and $33.5 \pm 15.5 \mathrm{~mL} / \mathrm{m}^{2}$, respectively (rho $=0.16, P=$ $.08, \mathrm{n}=20$ ). Using two echocardiographic imaging planes (Figure 3, B), the echocardiographic and MRI volumes were $21.1 \pm 7.5 \mathrm{~mL} / \mathrm{m}^{2}$ and $30.6 \pm 13.6 \mathrm{~mL} / \mathrm{m}^{2}$, respectively (rho $=0.2, P=.3, \mathrm{n}=8$ ).

The other anatomic measurements by echocardiography and MRI are shown in Table 1. MV annulus z-score in the echocardiographic 4-chamber view was an independent predictor of LVEDV observed by MRI $(P=.02)$, whereas AV $\mathrm{z}$-score and LVEDd z-score were not.

\section{Potential LV Volumes by MRI}

Every patient had a bigger potential than observed LVEDV (Figure 4). The mean potential increase of LVEDV was $8.8 \%$ for patients with AS, $35.0 \%$ for children with AVSD, and $23.0 \%$ in patients with HLHC.

\section{Aortic Blood Flow and Shunt Volumes}

PCMRI data were available in 18 of 20 patients. The measurements and calculations of the PCMRI flow analysis are given in Table 2. Blood flow volume in the ascending aorta did not correlate with LVEDV by echocardiography or MRI (rho $=0.01, P=.7 ;$ rho $=0.04, P=.5$, respectively). All patients had antegrade flow in the AAO. In those with a subsequent $\mathrm{BV}$ repair, all of whom survived the early postoperative period, the AAO flow was $1.63 \pm 0.57 \mathrm{~L} \cdot \mathrm{min}^{-1} \cdot \mathrm{m}^{-2}$ with a minimum of $1.0 \pm \mathrm{L} \cdot \min ^{-1} \cdot \mathrm{m}^{-2}$ in one patient. There was no correlation between the AAO flow and AV annulus z-score (rho $=0.003, P=.83$ ), $\mathrm{MV}$ annulus z-score (rho $=0.02, P=0.69$ ), or AAO diameter z-score (rho $=$ $0.007, P=.74$ ) by echocardiography.

\section{Outcome}

Surgical decision-making was based on multiple factors, including the patients' age and weight, their clinical status, associated cardiovascular and other lesions, imaging, as well as parental preference. In particular, in the absence of other contraindications toward a BV repair, if the MRI showed an actual LVEDV of more than the previously published cutoff of $20 \mathrm{~mL} / \mathrm{m}^{2}$, we felt encouraged to pursue a BV strategy, especially if the potential LVEDV was significantly larger. Likewise, if the MRI demonstrated that the $\mathrm{LV}$ outflow tract was capable of handling more than $1.0 \mathrm{~L}$ $\cdot \min ^{-1} \cdot \mathrm{m}^{-2}$ preoperatively, it seemed likely that a sufficient cardiac output would pass through it after an intervention. In the third patient of this series, hemodynamic measurements were performed intraoperatively to confirm the adequacy for $\mathrm{BV}$ repair.

Eighteen of 20 children underwent surgery or an intervention. One patient (HLHC, $1.70 \mathrm{~kg}$, LVEDV $12.8 \mathrm{~mL} /$ $\mathrm{m}^{2}$ by MRI) was discharged home under compassionate care by parental choice and another patient (HLHC, 2.30 $\mathrm{kg}$, LVEDV by MRI $30.8 \mathrm{~mL} / \mathrm{m}^{2}, \mathrm{LV}$ and $\mathrm{RV}$ ejection 


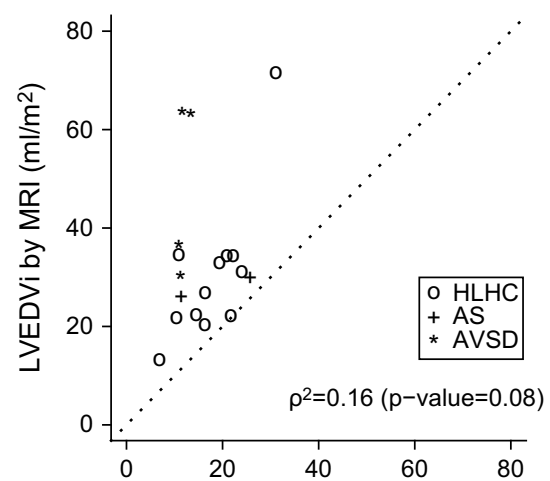

A LVEDVi by echo $\mathrm{mp}$ Simpson's $\left(\mathrm{ml} / \mathrm{m}^{2}\right)$ $\mathrm{n}=20$

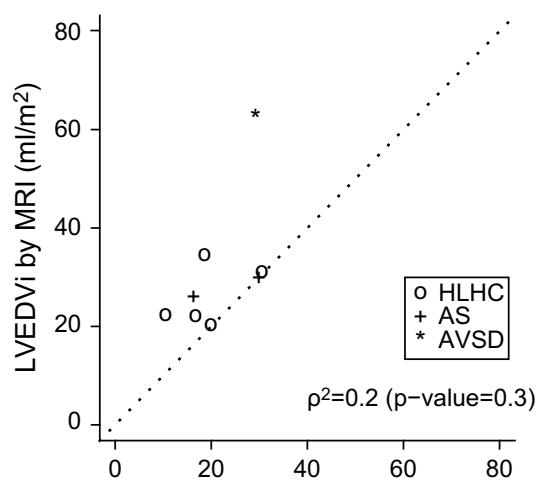

B LVEDVi by echo bp Simpson's $\left(\mathrm{ml} / \mathrm{m}^{2}\right)$

$n=8$

FIGURE 3. Left ventricular end-diastolic volume ( $L V E D V)$ measured by echocardiography versus magnetic resonance imaging (MRI), indexed to body surface area. A, Echocardiographic volume measurements, using the monoplane ( $m p$ ) Simpson method from an apical 4-chamber view. B, Biplane ( $b p$ ) echocardiographic volumetry from the apical 4-chamber and 2-chamber views. AS, Aortic stenosis; AVSD, atrioventricular septal defect; HLHC, hypoplastic left heart complex.

fractions $18 \%$ and $29 \%$, respectively) died without an intervention at 6 weeks of age owing to ventricular dysfunction and periventricular leukomalacia diagnosed on day 2 after birth that precluded heart transplantation. Two patients were palliated with a UV strategy: one patient with critical AS, an MRI LVEDV of $44.1 \mathrm{~mL} / \mathrm{m}^{2}$, but decreased ejection fraction, endocardial fibroelastosis, and a small and dysplastic MV (z-score -2.3); the other with an unbalanced AVSD, an LVEDV of $20.7 \mathrm{~mL} / \mathrm{m}^{2}$, LV outflow tract obstruction, a single left papillary muscle, and decreased ventricular function with an ejection fraction of $44 \%$ (Figure 1). This patient eventually underwent a hybrid procedure (surgical pulmonary artery banding and ductal stenting in the catheter laboratory) at 3 weeks of age and a Damus-Kaye-Stansel anastomosis and bidirectional cavopulmonary anastomosis at 7 months. Postoperatively, central pulmonary artery obstruction led to a cardiac arrest followed by a cerebral ischemic injury and death. Two patients with critical AS underwent balloon valvuloplasty.

TABLE 1. Comparison of echocardiographic and magnetic resonance imaging measurements (mean \pm standard deviations)

\begin{tabular}{lccl}
\hline & Echocardiography & MRI & $P$ value \\
\hline LVEDV bp $\left(\mathrm{mL} / \mathrm{m}^{2}\right)$ & $21.1 \pm 7.5$ & $30.6 \pm 13.6$ & 0.3 \\
LVEDV mp $\left(\mathrm{mL} / \mathrm{m}^{2}\right)$ & $16.0 \pm 7.0$ & $33.5 \pm 15.5$ & 0.08 \\
AV annulus & $4.9 \pm 1.0$ & $5.9 \pm 1.0$ & 0.006 \\
MV annulus & $7.5 \pm 1.5$ & $8.9 \pm 1.2$ & 0.01 \\
TV annulus & $12.8 \pm 2.3$ & $14.1 \pm 2.3$ & 0.14 \\
AAO (mm) & $6.5 \pm 1.4$ & $5.9 \pm 1.2$ & 0.4 \\
Transverse arch $(\mathrm{mm})$ & $3.5 \pm 0.9$ & $3.6 \pm 1.1$ & 0.9 \\
Isthmus $(\mathrm{mm})$ & $3.2 \pm 1.1$ & $3.3 \pm 1.1$ & 0.6 \\
\hline
\end{tabular}

$A A O$, Ascending aorta; $A V$, aortic valve; $b p$, biplane; $L V E D V$, left ventricular end-diastolic volume; $m p$, monoplane; $M R I$, magnetic resonance imaging; $M V$, mitral valve; $T V$, tricuspid valve.
The remaining 16 patients underwent a BV approach. There was no early postoperative mortality. One patient died at the age of 4 months (AVSD, LVEDV $29.1 \mathrm{~mL} / \mathrm{m}^{2}$ by MRI) of aspiration pneumonia. Both patients with critical AS, who underwent BV repair with balloon valvuloplasty as their first intervention, survived after balloon valvuloplasty. One of them had previously undergone an arch repair. Neither required a reintervention after the balloon valvuloplasty. According to the original ${ }^{10}$ and the recently revised score ${ }^{11}$ by the Congenital Heart Surgeons Society, only one of them was predicted to have a 5-year survival advantage with this strategy (5.8\% and $6.7 \%$, respectively). In the other patient, the original and the revised models estimated $2.9 \%$ and $15.6 \% 5$-year survival benefits with a UV approach. Applying the Rhodes score, both of these patients would have been subject to a UV strategy. ${ }^{12}$ They also had an aortic root size less than $3.5 \mathrm{~mm} / \mathrm{m}^{2}$ and an MV annulus area less than $4.75 \mathrm{~cm}^{2} / \mathrm{m}^{2}$, both indicating a bad prognosis after BV repair. ${ }^{12}$ Merely the LV length/ratio predicted a successful $\mathrm{BV}$ repair in both of them. An echocardiographic LVEDV greater than $20 \mathrm{~mL} / \mathrm{m}^{2}$, measured by biplane Simpson, was present in 1 of the 2 patients with AS and 3 of 7 total survivors with a BV repair. ${ }^{13}$

Two patients (one with HLHC and one with AVSD) underwent MV repair as a secondary procedure; the AVSD patient eventually required an MV prosthesis. One patient with HLHC received subaortic fibromyectomy 13 months after the initial arch reconstruction. There were no operative deaths in either the UV or BV groups. Of the 16 patients after $\mathrm{BV}$ repair, 1 died at the age of 4 months (AVSD, LVEDV $29.1 \mathrm{~mL} / \mathrm{m}^{2}$ by MRI) of aspiration pneumonia. There was 1 late death in the UV repair group following the second stage of the repair.

The postoperative growth for the AV, MV, and LVEDd as monitored by echocardiography is presented in Figure 5. 


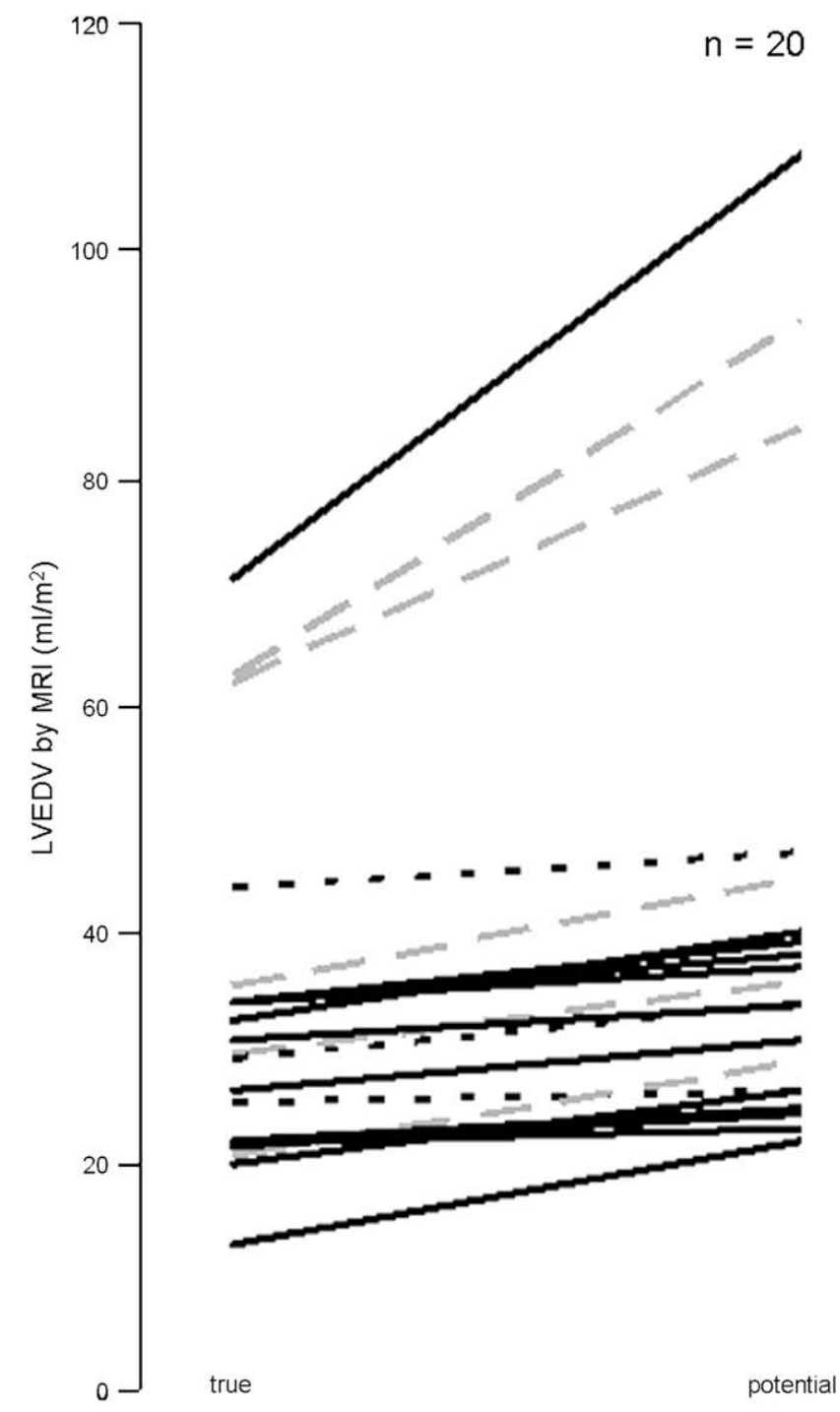

FIGURE 4. Potential left ventricular end-diastolic volumes ( $L V E D V)$ by magnetic resonance imaging (MRI) compared with measured true preoperative volumes. Dotted black lines, Patients with critical aortic stenosis; broken gray lines, patients with unbalanced atrioventricular septal defect; solid black lines, patients with hypoplastic left heart complex.

\section{DISCUSSION}

Predictors of early postoperative outcome must address whether the LV, after BV repair, is able to generate enough cardiac output to sustain the systemic circulation. Nearly all published predictors, however, are based on 2-dimensional anatomic rather than functional, indices. ${ }^{13-16}$ At our institution, there are two indications for cardiac catheterization in patients with marginally small LVs: One is to outline anatomy that cannot be detailed by echocardiography (or MRI), which was not the case in any of the patients presented here. The second is to perform an intervention, such as aortic valvuloplasty or ductal stenting during a hybrid procedure. Ideally, however, only unequivocal candidates for an inter-
TABLE 2. Flow measurements and shunt calculations

\begin{tabular}{|c|c|c|}
\hline & Mean & SD \\
\hline AAO flow $\left(\mathrm{L} \cdot \mathrm{min}^{-1} \cdot \mathrm{m}^{-2}\right)$ & 1.53 & 0.54 \\
\hline rSVC flow $\left(\mathrm{L} \cdot \min ^{-1} \cdot \mathrm{m}^{-2}\right)$ & 1.16 & 0.56 \\
\hline ISVC flow $\left(\mathrm{L} \cdot \min ^{-1} \cdot \mathrm{m}^{-2}\right)$ & 0.05 & 0.18 \\
\hline DAO flow $\left(\mathrm{L} \cdot \mathrm{min}^{-1} \cdot \mathrm{m}^{-2}\right)$ & 0.98 & 0.42 \\
\hline RPA flow $\left(\mathrm{L} \cdot \min ^{-1} \cdot \mathrm{m}^{-2}\right)$ & 3.07 & 1.40 \\
\hline LPA flow $\left(\mathrm{L} \cdot \min ^{-1} \cdot \mathrm{m}^{-2}\right)$ & 2.14 & 1.12 \\
\hline Qp (RPA flow + LPA flow $)\left(\mathrm{L} \cdot \mathrm{min}^{-1} \cdot \mathrm{m}^{-2}\right)$ & 5.21 & 2.44 \\
\hline Qs $($ SVC flow + DAO flow $)\left(\mathrm{L} \cdot \mathrm{min}^{-1} \cdot \mathrm{m}^{-2}\right)$ & 2.18 & 0.83 \\
\hline Qp/Qs & 2.86 & 1.81 \\
\hline Total left-to-right shunt $\left(\mathrm{L} \cdot \min ^{-1} \cdot \mathrm{m}^{-2}\right)^{*}$ & 3.04 & 2.78 \\
\hline PDA flow (left to right) $\left(\mathrm{L} \cdot \min ^{-1} \cdot \mathrm{m}^{-2}\right)$ & -0.46 & 0.95 \\
\hline Intracardiac left-to-right shunt $\left(\mathrm{L} \cdot \min ^{-1} \cdot \mathrm{m}^{-2}\right) \dagger$ & 3.51 & 2.39 \\
\hline
\end{tabular}

vention should undergo cardiac catheterization. The difficulty in establishing a uniformly recognized predictor of outcome after surgery, along with the observation that it is not always the patients with the smallest LV structures that have an unsuccessful BV repair, suggest that a different approach is needed. ${ }^{2}$ In this study, we attempted to overcome these limitations and compress the size of the marginal decision-making zone within the spectrum of borderline hypoplasia of the LV by using 3-dimensional anatomic and functional parameters of native LV performance. This study is the first to show that functional MRI in these neonatal patients is feasible and may be useful.

\section{Output as the Result of Size and Function}

Cardiac output is the result of a complex interplay of heart rate, LV preload, ventricular interaction, myocardial contractility, and systemic resistance. Notwithstanding the ductal contribution to the systemic circulation, the presence of forward flow in the ascending aorta, seen echocardiographically, is reassuring when BV repair is considered. ${ }^{17}$ Our group has recently shown that a systemic flow of only $1.8 \pm 0.6 \mathrm{~L} \cdot \mathrm{min}^{-1} \cdot \mathrm{m}^{-2}$ was present in survivors early after the Norwood operation. ${ }^{18}$ The survivor with a BV repair in our cohort demonstrated preoperatively that a flow comparable with that number $\left(1.63 \pm 0.57 \mathrm{~L} \cdot \mathrm{min}^{-1} \cdot \mathrm{m}^{-2}\right)$ could pass through the $\mathrm{LV}$ outflow tract and $\mathrm{AAO}$, with a minimum flow as low as $1.0 \mathrm{~L} \cdot \mathrm{min}^{-1} \cdot \mathrm{m}^{-2}$. Therefore, although our data cannot provide a threshold below which preinterventional aortic flow may be inadequate, we chose $1.0 \mathrm{~L}$. $\min ^{-1} \cdot \mathrm{m}^{-2}$ pragmatically in this series.

PCMRI is the method of choice for flow volume measurements. ${ }^{19,20}$ If the region of interest contains at least 16 pixels, the inaccuracy is less than $10 \% .^{21,22}$ In our study, the smallest aortic cross-sectional area was $13 \mathrm{~mm}^{2}$, seen in the smallest neonate, weighing $1.70 \mathrm{~kg}$. In this child, the region of 

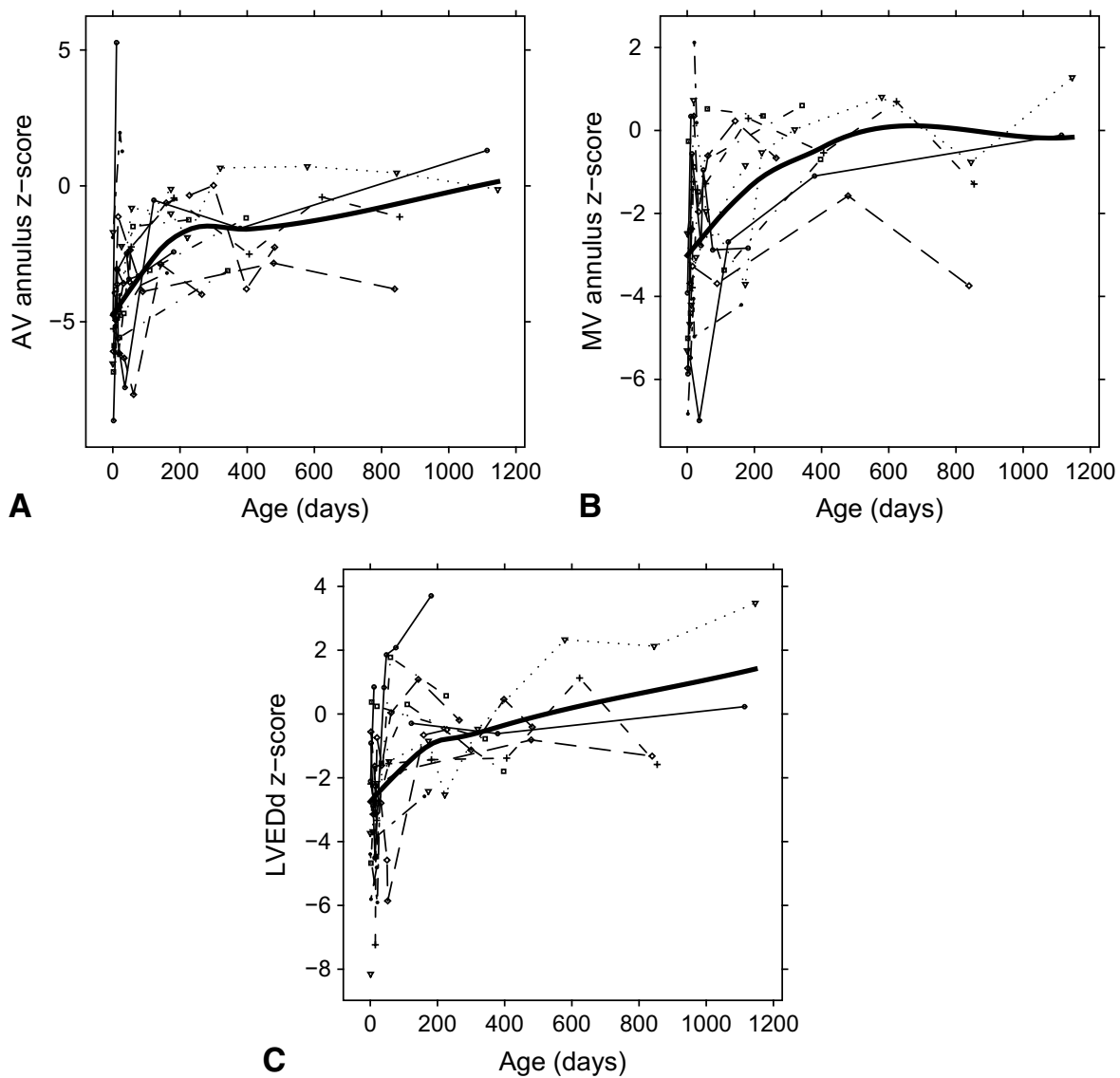

FIGURE 5. Postoperative growth after biventricular repair, monitored by serial echocardiograms: The first data point is the preoperative size; all subsequent points are postoperative measurements. Z-scores for (A) aortic valve annulus, (B) mitral valve annulus (excluding patients with atrioventricular septal defect), and $(C)$ left ventricular end-diastolic diameter.

interest contained 28 pixels at a spatial resolution of $1.00 \times$ $0.47 \mathrm{~mm}$. This report is the first of noninvasive shunt calculations in neonates. ${ }^{23,24} \mathrm{We}$ recently assessed the accuracy of PCMRI in normal adult volunteers, using the same magnet as for this study, and found an error of only $0.02 \pm 0.2$ $\mathrm{L} \cdot \min ^{-1} \cdot \mathrm{m}^{-2}$ when comparing the measured flow through the $\mathrm{DAO}$ with the measured flow (AAO-superior vena cava flow). This deviation was equivalent to $0.69 \% \pm 5.96 \%$ of systemic flow (H. W. Goo, MD, unpublished data, 2007). In the present patient cohort, characterized by a labile balance of the pulmonary and systemic blood flows, it is important to obtain flow data from various locations as quickly as possible and to keep the conditions for continuous breathing or ventilation and the patient's fluid status as constant as possible.

\section{Echocardiography Underestimates LV Volume}

In older patients, MRI is the established gold standard for ventricular volumetry inasmuch as it does not rely on a presumed shape of the ventricle, in contrast to the echocardiographic Simpson method that assumes a bullet-shaped LV, with a circular cross section. ${ }^{5,25-29}$ The fact that the shape of the LV in patients with a borderline LV is abnormal is almost certainly the reason for the inaccuracy of this technique, when compared with MRI (Figure 3). ${ }^{30}$ This error leads to an underestimation of the true volume if the septum is bowing toward the left side, which is amplified when the LV diameter is measured only in the 4-chamber plane instead of a biplane assessment (Figure 3). None of the patients received an MRI and an echocardiogram on the same day. In some of them, the two tests were 2 weeks apart. This, through ventricular growth and altered shunts and loading conditions, might have contributed to the discrepancy between echocardiography and MRI-derived volumetrics. MV annulus size predicts survival in patients with left-sided obstructive lesions. ${ }^{12,31,32}$ In a report by Schwartz, Gauvreau, Geva, ${ }^{31}$ echocardiographic MV annulus diameter and LVEDV correlated in children with multiple left-sided obstructive lesions (rho $=0.47, P<.001$ ). Our data confirm that echocardiographic measurements of LV inflow, but not outflow size or end-diastolic dimension, predict true LVEDV as measured by MRI.

MRI measurements of the AV and MV were significantly larger than echocardiographic measurements (Table 1). 
Echocardiography, especially in neonates who typically have very good ultrasound windows, appears superior to MRI for intracardiac structures, owing to better temporal resolution and tissue/blood contrast in echocardiography versus white blood MRI.

\section{MRI Changes Surgical Decision-making}

Conversion to a UV circulation after a failed BV repair carries a high mortality, whereas the opposite sequence of interventions appears to have a better result. ${ }^{1,12,33} \mathrm{~A} \mathrm{UV}$ circulation, on the other hand, may limit the child's functional outcome. The hitherto largest study by the Congenital Heart Surgeons Society estimated that half of all patients with critical AS who underwent a primary BV repair should have had a Norwood procedure, whereas $20 \%$ of those who did have a UV repair would probably have survived after BV repair. ${ }^{10}$ The Society's original score to predict 5-year survival benefits of UV over BV repair was recently revised. ${ }^{11}$ In both the original and the new version, 1 of 2 patients with AS and a successful BV repair in this cohort would not have been given that chance.

Concluding that the adverse effects of inflow and outflow hypoplasia and a small LV are cumulative, Rhodes and colleagues ${ }^{12}$ combined the diameters of the aortic root and MV annulus and the LV length in a score to predict survival after BV repair in patients with AS. In our cohort, it would have mandated a Norwood operation in both patients with AS who underwent successful BV correction.

The Rhodes score, however, is not applicable to patients with HLHC or unbalanced AVSD. ${ }^{34}$ In these patient groups, discriminating parameters are scarce, and although several reports of survivors of BV repairs with smaller ventricles undermine this cutoff, an LVEDV of at least $20 \mathrm{~mL} / \mathrm{m}^{2}$, measured by echocardiography or x-ray angiography, continues to be commonly used as a prerequisite for BV repair. ${ }^{31,35}$ Interestingly, these studies used echocardiography for their volumetry, whereas the threshold of $20 \mathrm{~mL} / \mathrm{m}^{2}$ was originally established angiographically. ${ }^{36}$ In our group of patients with $\mathrm{BV}$ repair, none of whom died perioperatively, the smallest LVEDV was $20 \mathrm{~mL} / \mathrm{m}^{2}$ by MRI. More than half of them would have undergone UV repair, applying this still widely used criterion to their echocardiographic measurements. Thus our data suggest that an LVEDV of $20 \mathrm{~mL} /$ $\mathrm{m}^{2}$ or more is sufficient to undergo BV repair whereas echocardiographically obtained volumes are less meaningful and may be considerably smaller.

In patients with unbalanced AVSD or HLHC, who have $\mathrm{RV}$ pressure and/or volume overload, a mere expansion of the LV can lead to a substantial increase in volume, as expressed by a large difference between the true and the potential LVEDV (Figures 2 and 4). ${ }^{3,37}$ In patients with an actual LVEDV of $20 \mathrm{~mL} / \mathrm{m}^{2}$ or less but otherwise encouraging anatomy and function, a large potential increase in ventricular size after optimized filling conditions is reassuring toward a BV repair. In contrast, in critical AS, which is characterized by a markedly increased afterload, the LV retains a near ideal shape. Therefore, the potential volume increase in these patients is small, even if RV and LV loading conditions are optimized. Expansion and an altered shape of the LV immediately postoperatively, as well as continued growth, presumably contribute to the increase in size of LV structures after BV repair (Figure 5).

Of note, 2 of our patients underwent UV repair despite an LVEDV greater than $20 \mathrm{~mL} / \mathrm{m}^{2}$, because valvular anatomy and/or myocardial function at the time of surgery was deemed inadequate for a BV approach, underscoring the continued importance of associated lesions, especially of the $\mathrm{LV}$ inflow. Han and colleagues ${ }^{38}$ recently showed that after neonatal intervention for critical AV stenosis the potential of the MV annulus for catch-up growth is smaller than that of the AV and LV diameter.

It is important to appreciate that long-term survival does not necessarily exclusively reflect appropriate selection, as continuing growth and physiologic remodeling are necessary in these BV repairs. Our early data and those of others regarding expansion and growth of LV structures are reassuring in this regard, but careful follow-up will be required. $^{38}$

The ideal outcome for any patient is a serial circulation using two ventricles, free of repeat interventions and late complications. The recent analysis of 362 patients with critical AS by the Congenital Heart Surgeons Society suggests that $56 \%$ of patients who are submitted to a BV repair would have had a long-term survival advantage from a UV approach, and that erring on the side of a BV repair has disastrous effects in a large proportion of these patients. ${ }^{11}$

\section{LIMITATIONS}

The principal limitation of this study is the relatively small number of patients, making it difficult to test for statistical significance. Therefore, a lack of an association in our analysis does not exclude it. Cardiac MRI is now part of the standard of care in patients with borderline small LVs in whom there is doubt whether to attempt BV or UV at our institution. The present study contains a selection bias inasmuch as not all patients with comparable pathologic anatomy underwent an MRI in the early phase of this study.

This study was not designed to look at outcome for particular lesions or interventions. The patients were selected on the basis of having undergone a preoperative MRI to assess LV size. There were no perioperative deaths after the initial BV repair and no patient had to be converted into a UV circulation. Therefore, MRI-derived risk factors for unsuccessful BV repair could not be isolated from our data. Prospective studies of a greater cohort with more adverse outcomes after BV repair are needed to answer this question. 
A biplane echocardiographic volume assessment in all patients would have been desirable as it is the most common method and more accurate than a monoplane assessment.

Early postoperative MRI examinations are desirable to detail how much of the potential volume is actually recruited by loading the LV and unloading the RV.

In conclusion, MRI volumetric and flow measurements in neonates with borderline hypoplasia of the LV are feasible. Echocardiographic volumetry underestimates LV volume, and indices based on anatomy alone may not adequately predict survival after BV repair. Consequently, assessment of the LV volume and cardiac output by MRI may change surgical decision-making regarding UV versus BV repair.

\section{References}

1. Noonan JA, Nadas AS. The hypoplastic left heart syndrome: an analysis of 101 cases. Pediatr Clin North Am. 1958;5:1029-56.

2. Tchervenkov CI, Tahta SA, Jutras LC, Beland MJ. Biventricular repair in neonates with hypoplastic left heart complex. Ann Thorac Surg. 1998;66:1350-7.

3. Phoon CK, Silverman NH. Conditions with right ventricular pressure and volume overload, and a small left ventricle: 'hypoplastic"' left ventricle or simply a squashed ventricle? J Am Coll Cardiol. 1997;30:1547-53.

4. Daubeney PE, Blackstone EH, Weintraub RG, Slavik Z, Scanlon J, Webber SA. Relationship of the dimension of cardiac structures to body size: an echocardiographic study in normal infants and children. Cardiol Young. 1999;9:402-10.

5. Silverman NH, McElhinney DB. Echocardiography of hypoplastic ventricles. Ann Thorac Surg. 1998;66:627-33.

6. Cleveland WS, Grosse E, Shyu MJ. Local regression models. In: Chambers JM, Hastie T, eds. Statistical models in S. New York: Chapman and Hall; 1992. p. 309-76.

7. R Foundation for Statistical Computing: http://www.r-project.org [computer program]. Vienna, Austria: Department of Statistics and Mathematics, WU, Vienna, Austria. 2007.

8. Harrell FE Jr. Design: Design Package. R package version 2.0-12 [computer program]. Department of Biostatistics, Vanderbilt University, Nashville [TN]; 2005.

9. Pinheiro J, Bates D, DeloRoy S, Sakar D, nlme. Linear and non-linear mixed effect models [computer program]. Department of Statistics and Mathematics, WU, Vienna, Austria; 2007.

10. Lofland GK, McCrindle BW, Williams WG, Blackstone EH, Tchervenkov CI, Sittiwangkul R, et al. Critical aortic stenosis in the neonate: a multi-institutional study of management, outcomes, and risk factors. Congenital Heart Surgeons Society. J Thorac Cardiovasc Surg. 2001;121:10-27.

11. Hickey EJ, Caldarone CA, Blackstone EH, Lofland GK, Yeh T Jr, Pizarro C, et al. Critical left ventricular outflow tract obstruction: the disproportionate impact of biventricular repair in borderline cases. J Thorac Cardiovasc Surg. 2007;134: 1429-36.

12. Rhodes LA, Colan SD, Perry SB, Jonas RA, Sanders SP. Predictors of survival in neonates with critical aortic stenosis. Circulation. 1991;84:2325-35.

13. Hammon JW Jr, Lupinetti FM, Maples MD, Merrill WH, First WH, Graham TP Jr, et al. Predictors of operative mortality in critical valvular aortic stenosis presenting in infancy. Ann Thorac Surg. 1988;45:537-40.

14. Colan SD, McElhinney DB, Crawford EC, Keane JF, Lock JE. Validation and reevaluation of a discriminant model predicting anatomic suitability for biventricular repair in neonates with aortic stenosis. J Am Coll Cardiol. 2006;47:1858-65.

15. Alboliras ET, Mavroudis C, Pahl E, Gidding SS, Backer CL, Rocchini AP. Left ventricular growth in selected hypoplastic left ventricles: outcome after repair of coarctation of aorta. Ann Thorac Surg. 1999;68:549-55.

16. Cohen MS, Rychik J. The small left ventricle: how small is too small for biventricular repair? Semin Thorac Cardiovasc Surg Pediatr Card Surg Annu. 1999; 2:189-202

17. Kovalchin JP, Brook MM, Rosenthal GL, Suda K, Hoffman JI, Silverman NH. Echocardiographic hemodynamic and morphometric predictors of survival after two-ventricle repair in infants with critical aortic stenosis. J Am Coll Cardiol. 1998;32:237-44.

18. Li J, Zhang G, McCrindle BW, Holtby H, Humpl T, Cai S, et al. Profiles of hemodynamics and oxygen transport derived by using continuous measured oxygen consumption after the Norwood procedure. J Thorac Cardiovasc Surg. 2007;133: 441-8.

19. Lotz J, Meier C, Leppert A, Galanski M. Cardiovascular flow measurement with phase-contrast MR imaging: basic facts and implementation. Radiographics. 2002;22:651-71.

20. Powell A, Geva T. Blood flow measurement by magnetic resonance imaging in congenital heart disease. Pediatr Cardiol. 2000;21:47-58.

21. Hofman MB, Visser FC, van Rossum AC, Vink QM, Sprenger M, Westerhof N. In vivo validation of magnetic resonance blood volume flow measurements with limited spatial resolution in small vessels. Magn Reson Med. 1995;33: 778-84.

22. Greil G, Geva T, Maier SE, Powell AJ. Effect of acquisition parameters on the accuracy of velocity encoded cine magnetic resonance imaging blood flow measurements. J Magn Reson Imaging. 2002;15:47-54.

23. Petersen SE, Voigtlander T, Kreitner KF, Kalden P, Wittlinger T, Scharhag J, et al. Quantification of shunt volumes in congenital heart diseases using a breath-hold MR phase contrast technique-comparison with oximetry. Int J Cardiovasc Imaging. 2002;18:53-60.

24. Beerbaum P, Korperich H, Barth P, Esdorn H, Gieseke J, Meyer H. Noninvasive quantification of left-to-right shunt in pediatric patients: phase-contrast cine magnetic resonance imaging compared with invasive oximetry. Circulation. 2001; 103:2476-82.

25. Mogelvang J, Stokholm KH, Saunamaki K, Reimer A, Stubgaard M, Thomsen C, et al. Assessment of left ventricular volumes by magnetic resonance in comparison with radionuclide angiography, contrast angiography and echocardiography. Eur Heart J. 1992;13:1677-83.

26. Kiss K, Varghese A, Stiskal M, Czembirek H, Mlczoch J, Pennell DJ. Cardiovascular magnetic resonance: current clinical practice and future potential. Semin Thorac Cardiovasc Surg. 2004;16:235-41.

27. Alfakih K, Reid S, Jones T, Sivananthan M. Assessment of ventricular function and mass by cardiac magnetic resonance imaging. Eur Radiol. 2004;14:1813-22.

28. Pons-Llado G. Assessment of cardiac function by CMR. Eur Radiol. 2005; 15(Suppl. 2):B23-32.

29. Debatin JF, Nadel SN, Paolini JF, Sostman HD, Coleman RE, Evans AJ, et al. Cardiac ejection fraction: phantom study comparing cine MR imaging, radionuclide blood pool imaging, and ventriculography. J Magn Reson Imaging. 1992; 2:135-42.

30. Boudoulas H, Ruff PD, Fulkerson PK, Lewis RP. Relationship of angiographic and echographic dimensions in chronic left ventricular dilatation. Am Heart J. 1983;106:356-62.

31. Schwartz ML, Gauvreau K, Geva T. Predictors of outcome of biventricular repair in infants with multiple left heart obstructive lesions. Circulation. 2001;104: 682-7.

32. Pelech AN, Dyck JD, Trusler GA, Williams WG, Olley PM, Rowe RD, et al. Critical aortic stenosis: survival and management. J Thorac Cardiovasc Surg. 1987; 94:510-7.

33. Pearl JM, Cripe LW, Manning PB. Biventricular repair after Norwood palliation. Ann Thorac Surg. 2003;75:132-6.

34. Tani LY, Minich LL, Pagotto LT, Shaddy RE, McGough EC, Hawkins JA. Left heart hypoplasia and neonatal aortic arch obstruction: is the Rhodes left ventricular adequacy score applicable? J Thorac Cardiovasc Surg. 1999;118:81-6.

35. Serraf A, Piot JD, Bonnet N, Lacour-Gayet F, Touchot A, Bruniaux J, et al. Biventricular repair approach in ducto-dependent neonates with hypoplastic but morphologically normal left ventricle. J Am Coll Cardiol. 1999;33:827-34.

36. Zeevi B, Keane JF, Castaneda AR, Perry SB, Lock JE. Neonatal critical valvar aortic stenosis. A comparison of surgical and balloon dilation therapy. Circulation. 1989;80:831-9.

37. van Son JA, Phoon CK, Silverman NH, Haas GS. Predicting feasibility of biventricular repair of right-dominant unbalanced atrioventricular canal. Ann Thorac Surg. 1997;63:1657-63.

38. Han RK, Gurofsky RC, Lee KJ, Dipchand AI, Williams WG, Smallhorn JF, et al. Outcome and growth potential of left heart structures after neonatal intervention for aortic valve stenosis. J Am Coll Cardiol. 2007;50:2406-14. 